\title{
Comparison of ultrasound fetal biometry of singleton fetuses with a reference chart in pregnant women with normal nutritional and health status
}

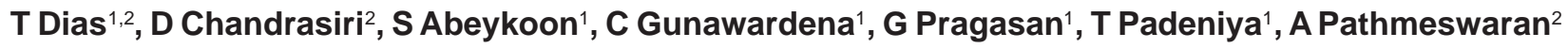

\begin{abstract}
Objectives The aim of this study was to compare fetal biometry of singleton fetuses in pregnant women with normal nutritional and health status in the Ampara district, with a commonly used reference chart.

Methods A cross sectional study was carried out in the Ampara District. Women with normal nutritional and health status and minimal environmental constraints on fetal growth ( $n=714)$ were enrolled during the first trimester and gestational age was confirmed by fetal crown-rump length measurement between 11 weeks + 0 days and 13 weeks + 6 days. For this study, each mother was considered only once for measurement of fetal biometry, at gestations between 11 and 41 weeks. Fetal bi-parietal diameter (BPD), head circumference $(\mathrm{HC})$, abdominal circumference $(\mathrm{AC})$ and femur length (FL) were measured using standard techniques, and separate regression models were fitted to estimate the means and standard deviations and derive gestation specific centiles for each parameter, assuming that the measurements have a normal distribution at each gestational age. The fetal biometry results obtained from the current study were compared with a commonly used reference chart.

Results The fitted 10th, 50th and 90th centiles at 40 weeks of gestation were, $87.9 \mathrm{~mm}, 93.2 \mathrm{~mm}$ and $98.5 \mathrm{~mm}$ for BPD, $313.8 \mathrm{~mm}, 328.9 \mathrm{~mm}$ and $344.0 \mathrm{~mm}$ for HC; 298.2 $\mathrm{mm}, 322.5 \mathrm{~mm}$ and $346.9 \mathrm{~mm}$ for $\mathrm{AC}$ and $69.7 \mathrm{~mm}, 75.0$ $\mathrm{mm}$ and $80.2 \mathrm{~mm}$ for FL. When compared with the reference chart, significant differences of fetal biometry were seen in the third trimester but not in the second trimester.

Conclusions Ultrasound fetal biometry of singleton fetuses in pregnant women with normal nutritional and health status in the Ampara District were significantly different in the third trimester, from a routinely used reference chart.
\end{abstract}

Ceylon Medical Journal 2017; 62: 47-56

DOI: http://doi.org/10.4038/cmj.v62i1.8434

\section{Introduction}

Fetal biometry is routinely measured during pregnancy to assess the gestational age and fetal growth. Any reference should ideally be derived from data from the same population in which it is to be applied. However in the absence of such local or national references, regional or international references have to be used, but the regional or international reference should be from a population as similar as possible to the local population. It has been demonstrated that the choice of an appropriate reference chart is important, because if the reference used for identification of a fetus at risk has a low sensitivity and specificity, it could lead to inappropriate interpretations and conclusions which could result in inappropriate management [1].

Fetal bi-parietal diameter (BPD) was used in the past to estimate gestational age but later fetal head circumference (HC) was found to be better at estimating gestational age [2, 3]. Microcephaly can be defined as BPD and $\mathrm{HC}>3$ standard deviations (SD) below the mean for gestational age using Brazilian based reference ranges [4]. Small for gestational age (SGA) and large for gestational age (LGA) have been used to define abnormal fetal growth. Diagnosis of a SGA fetus relies on ultrasound measurement of fetal abdominal circumference or estimation of fetal weight [5]. SGA and LGA are commonly defined as a fetal abdominal circumference (AC) less than the 10th percentile and AC more than the 90th percentile respectively of a particular reference at a given gestational age [5]. Fetal femur length (FL) less than 3rd centile has been considered in skeletal dysplasia [6]. Many ultrasound-estimated fetal weight (EFW) formulae have been designed and these are based on different fetal biometric parameters (BPD, HC, AC and FL) [1, 7]. An accurate estimation of EFW is needed to determine viability as well as to monitor and manage SGA and LGA fetuses. Fetal weight can be indirectly estimated both clinically and radiologically [8].

${ }^{1}$ District General Hospital, Ampara, ${ }^{2}$ Faculty of Medicine University of Kelaniya, Sri Lanka.

Correspondence: TD, e-mail: <thiran_dias@yahoo.com>. Received 12 October 2015 and revised version accepted 22 April 2016.

This is an open-access article distributed under the terms of the Creative Commons Attribution License, which permits unrestricted use, distribution, and reproduction in any medium, provided the original author and source are credited. 
There has been no systematic evaluation of the validity of any international ultrasound fetal biometric charts available for use in Sri Lanka. The aim of this study was to compare fetal biometry of singleton fetuses in pregnant women in the Ampara District with normal body mass indices (BMI), normal nutritional and health status and minimal environmental constraints on fetal growth, with the reference charts developed by Chitty et al. in England in 1994 which are commonly used in Sri Lanka [9-12]. The charts developed by Chitty et al. were derived from a population of Western Europeans (75\%) and AfroCaribbeans (25\%). Therefore these charts may not be entirely applicable to other ethnic groups.

\section{Methods}

A cross sectional study was carried out in four Medical Officers of Health $(\mathrm{MOH})$ areas (Ampara, Damana, Mahaoya and Uhana) and the District General Hospital in the Ampara District between January 2013 and February 2014. We enrolled women who initiated antenatal care at each study site before 14 weeks of gestation. with normal BMI and normal nutritional and health status and minimal environmental constraints on fetal growth. All pregnancies with first trimester ultrasound Crown- Rump Length (CRL) dating were included [6]. Inclusion criteria of the study are described in a previous publication [28]. Approval was obtained from the Ethics Review Committee of Ampara District General Hospital and written informed consent was obtained from all participants.

Ultrasound scans were performed by a single, experienced, certified medical officer (the 4th author) with experience in routine ultrasound examinations. $\mathrm{BPD}, \mathrm{HC}, \mathrm{AC}$ and FL measurements were obtained according to the established guidelines [13]. All examinations were performed trans-abdominally using a "GE Logiq E” ultra sound scanner. Each mother was considered only once for measurement of fetal biometry, for the purpose of this study, at gestations between 11 and 40 weeks.

The routine practice in the antenatal clinics was to perform only a single fetal biometry measurement for each parameter (HC, BPD, AC and FL) rather than taking multiple measurements. Therefore to create a similar setting within our study as to what was being practiced routinely, it was decided to take a single fetal biometry measurement for each parameter rather than taking multiple measurements of the same parameter and obtaining the mean. The sonographer was not aware of the gestational age of the fetus.

Altman and Chitty's statistical methods were used to analyse the data [14]. Linear and polynomial regression models were fitted separately to the mean and standard deviation (SD) as functions of gestational age to identify the model with the best fit [14]. The gestational age was rounded to completed weeks of gestation. For each biometric parameter a separate regression model was fitted to estimate the mean and standard deviation at each gestational age. Centiles were derived from this mean and standard deviation, assuming that the measurements have a normal distribution at each gestational age. The centiles were obtained making the assumption that at each gestational age the measurements had a normal distribution.

\section{Results}

We screened 3216 women commencing antenatal care at 11 weeks and 0 days of gestation, of whom 714 (22.2\%) women fulfilling the inclusion criteria were enrolled. Characteristics of the study group are given in Table 1. Both the normal plot and the plot of the standardised residuals against gestation show that the data met the assumptions well (Figures 1 and 2). The fitted centiles of BPD, HC, AC and FL for each gestation are shown in tables 2, 3, 4 and 5 respectively. Tables 6-9 show the comparisons of the means and standard deviations of fetal BPD, HC, AC and FL measurements obtained by the current study, compared with the data obtained from England, which were used to develop their reference charts [9-12]. In the second trimester there were no significant differences between the local fetal biometry and the reference charts. However, during the third trimester all fetal biometry parameters were different from the reference charts (Tables 6-9)

\section{Discussion}

Knowledge of estimated fetal weight is of paramount importance to obstetricians as the birth weight is the single most important factor determining neonatal survival and it is also considered in deciding the time and mode of delivery [5, 15-17]. Inaccurate measurements can lead to erroneous detection of fetal growth restriction or macrosomia leading to unnecessary intervention, maternal anxiety and iatrogenic perinatal morbidity; or may lead to inaccurate identification of growth-restricted fetuses as normal [18].

In conclusion, our study has shown that ultrasound fetal biometry of singleton fetuses in pregnant women with normal nutritional and health status in the Ampara district were significantly different in the third trimester, from a routinely used reference chart.

The charts developed by Chitty et al. Show a systematic error which becomes greater and statistically significant towards term, when in fact, fetal biometry becomes more important in the management of the pregnancy [9-12, 14]. Fetal growth abnormalities are more common during the third trimester. Accurate interpretation of fetal growth is of paramount importance in clinical decision making especially to time the delivery. Erroneous interpretations of fetal growth may either over or under diagnose LGA and SGA. 

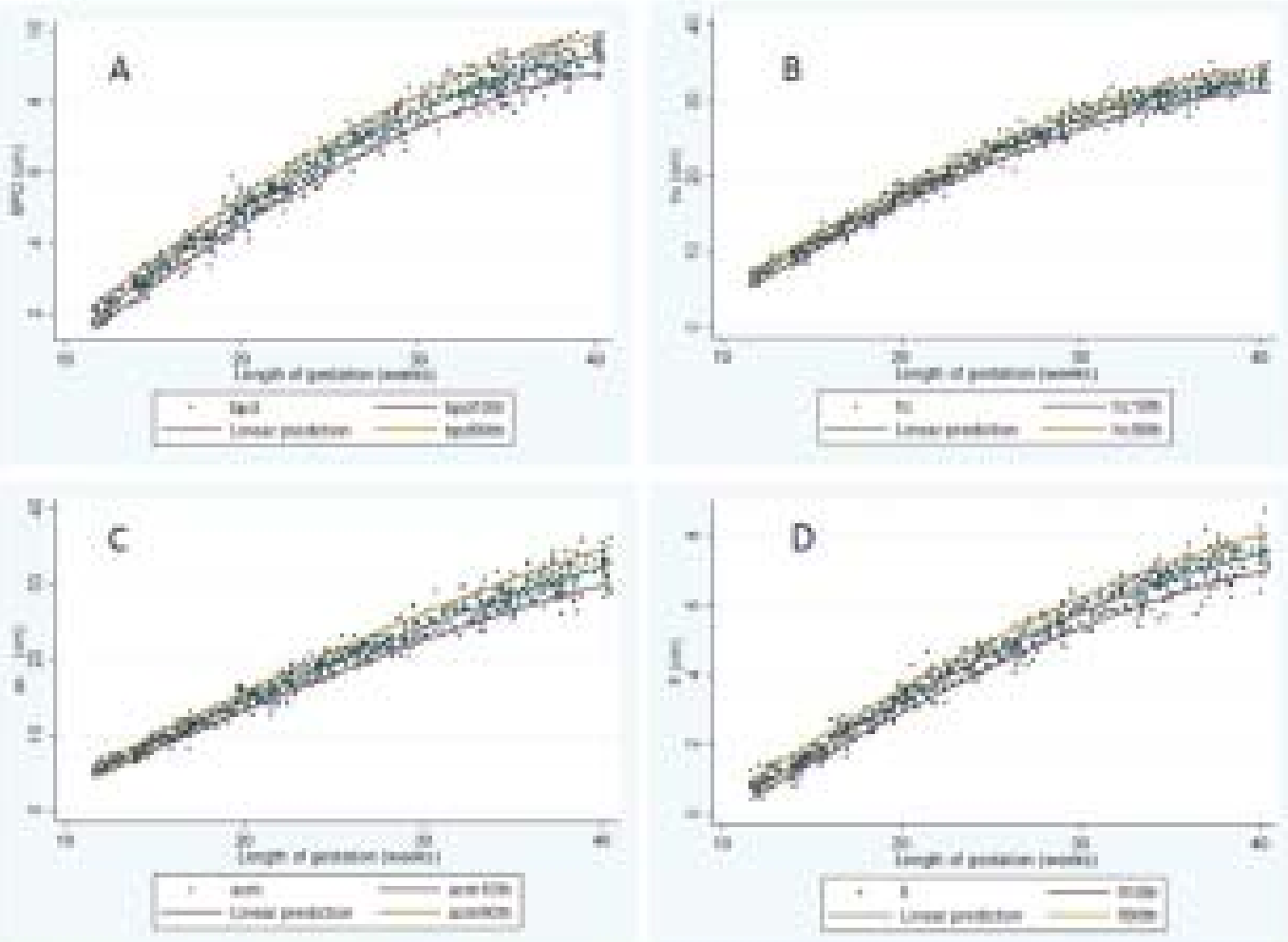

A: Bi Parietal Diameter, B: Head Circumference, C: Abdominal Circumference, D: Femur Length

Figure 1. Scatter plots for bi-parietal diameter, head circumference, abdominal circumference, and femur length with $10^{\text {th }}$ and $90^{\text {th }}$ centiles
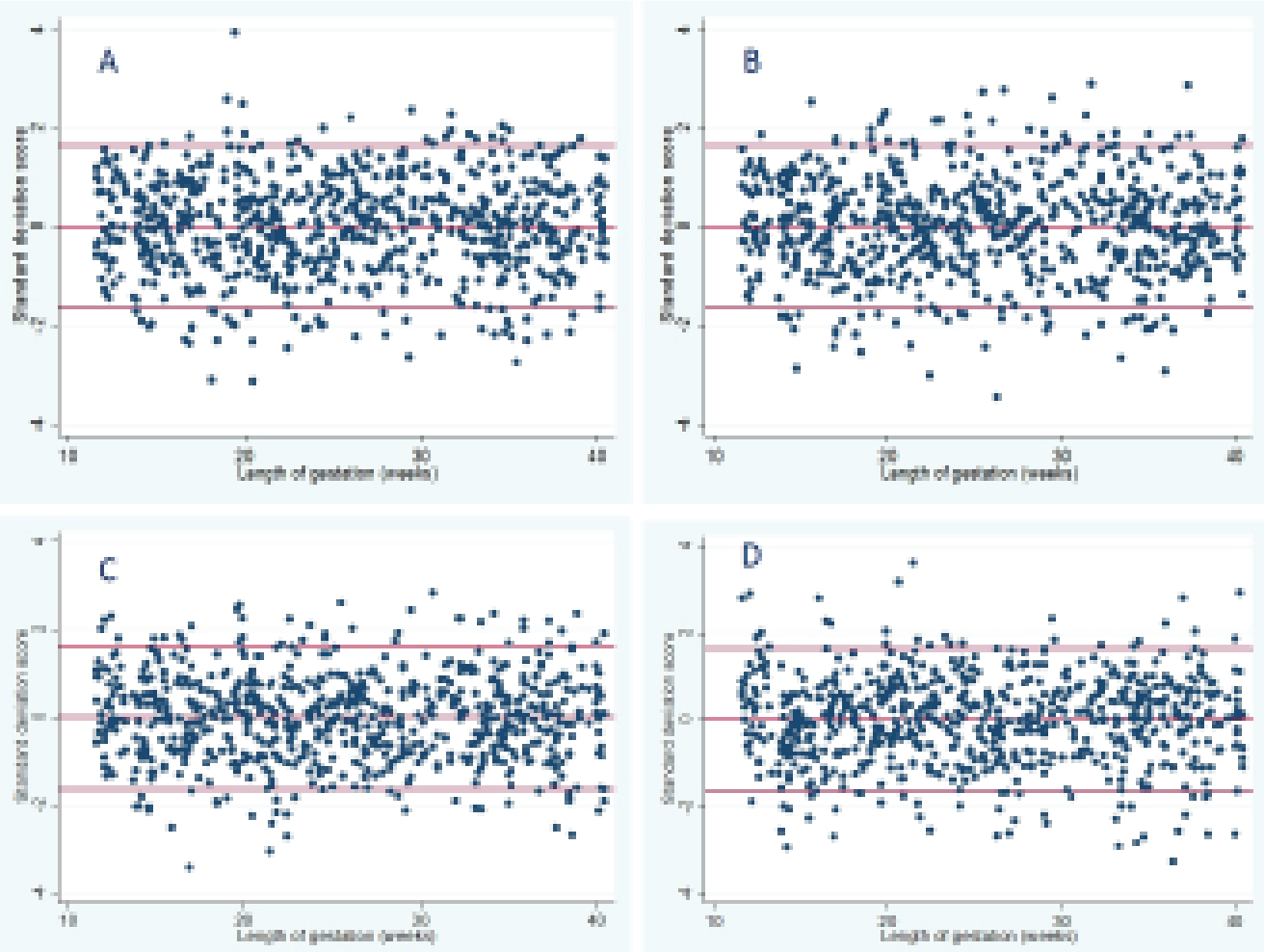

A: Bi Parietal Diameter, B: Head Circumference, C: Abdominal Circumference, D: Femur Length

Figure 2. Residual plots for bi-parietal diameter, head circumference, abdominal circumference, and femur length with $10^{\text {th }}$ and $90^{\text {th }}$ centiles 
Table 1. Characteristics of the study population

\begin{tabular}{|c|c|c|c|}
\hline & & Number (n) & Percent (\%) \\
\hline \multicolumn{4}{|l|}{ Ethnicity } \\
\hline & Sinhalese & 356 & 92 \\
\hline & Muslims & 23 & 6 \\
\hline & Tamils & 8 & 2 \\
\hline \multicolumn{4}{|l|}{ Parity } \\
\hline & $\mathrm{P} 1$ & 132 & 34 \\
\hline & P2 & 124 & 32 \\
\hline & P3 & 97 & 25 \\
\hline & P4 \& above & 34 & 9 \\
\hline \multicolumn{4}{|c|}{ Age : Range $=17$ to 42 years } \\
\hline & 1st Quartile & 23 & \\
\hline & Median & 27 & \\
\hline & 3rd Quartile & 31 & \\
\hline \multicolumn{4}{|c|}{$\mathrm{BMI}:$ Range $=18.5 \mathrm{Kgm}^{-2}$ to $25 \mathrm{Kgm}^{-2}$} \\
\hline & 1st Quartile & 19 & \\
\hline & Median & 21 & \\
\hline & 3rd Quartile & 23 & \\
\hline
\end{tabular}

Table 2. Fitted centiles for bi-parietal diameter in singleton fetuses of pregnant women, in Ampara district

\begin{tabular}{|c|c|c|c|c|c|c|}
\hline \multicolumn{7}{|c|}{ Centiles in millimeters } \\
\hline $\begin{array}{l}\text { Gestational } \\
\text { Age in } \\
\text { weeks }\end{array}$ & $\begin{array}{l}\text { 5th } \\
\text { centile }\end{array}$ & $\begin{array}{l}\text { 10th } \\
\text { centile }\end{array}$ & $\begin{array}{l}\text { 50th } \\
\text { centile }\end{array}$ & $\begin{array}{l}\text { 90th } \\
\text { centile }\end{array}$ & $\begin{array}{l}\text { 95th } \\
\text { centile }\end{array}$ & $\begin{array}{l}\text { Standard } \\
\text { deviation }\end{array}$ \\
\hline 12 & 16.1 & 17.1 & 20.4 & 23.7 & 24.7 & 2.6 \\
\hline 13 & 19.8 & 20.8 & 24.2 & 27.6 & 28.5 & 2.6 \\
\hline 14 & 23.5 & 24.5 & 27.9 & 31.4 & 32.4 & 2.7 \\
\hline 15 & 27.1 & 28.1 & 31.6 & 35.2 & 36.2 & 2.8 \\
\hline 16 & 30.6 & 31.6 & 35.2 & 38.8 & 39.9 & 2.8 \\
\hline 17 & 34.1 & 35.1 & 38.8 & 42.5 & 43.5 & 2.9 \\
\hline 18 & 37.5 & 38.6 & 42.3 & 46.0 & 47.1 & 2.9 \\
\hline 19 & 40.8 & 41.9 & 45.7 & 49.5 & 50.6 & 3.0 \\
\hline 20 & 44.1 & 45.2 & 49.1 & 52.9 & 54.0 & 3.0 \\
\hline 21 & 47.2 & 48.4 & 52.3 & 56.3 & 57.4 & 3.1 \\
\hline 22 & 50.3 & 51.5 & 55.5 & 59.5 & 60.6 & 3.1 \\
\hline 23 & 53.3 & 54.5 & 58.6 & 62.6 & 63.8 & 3.2 \\
\hline 24 & 56.2 & 57.4 & 61.5 & 65.7 & 66.9 & 3.2 \\
\hline 25 & 59.0 & 60.2 & 64.4 & 68.7 & 69.9 & 3.3 \\
\hline 26 & 61.7 & 62.9 & 67.2 & 71.5 & 72.7 & 3.4 \\
\hline 27 & 64.3 & 65.5 & 69.9 & 74.3 & 75.5 & 3.4 \\
\hline 28 & 66.8 & 68.0 & 72.5 & 76.9 & 78.2 & 3.5 \\
\hline 29 & 69.1 & 70.4 & 74.9 & 79.4 & 80.7 & 3.5 \\
\hline 30 & 71.4 & 72.7 & 77.3 & 81.8 & 83.1 & 3.6 \\
\hline 31 & 73.5 & 74.8 & 79.5 & 84.1 & 85.4 & 3.6 \\
\hline 32 & 75.5 & 76.9 & 81.6 & 86.3 & 87.6 & 3.7 \\
\hline 33 & 77.4 & 78.7 & 83.5 & 88.3 & 89.7 & 3.7 \\
\hline 34 & 79.1 & 80.5 & 85.3 & 90.2 & 91.6 & 3.8 \\
\hline 35 & 7 & 82.1 & 87.0 & 92.0 & 93.4 & 3.8 \\
\hline 36 & 82.2 & 83.6 & 88.6 & 93.6 & 95.0 & 3.9 \\
\hline 37 & 83.5 & 84.9 & 90.0 & 95.0 & 96.5 & 4.0 \\
\hline 38 & 84.6 & 86.1 & 91.2 & 96.3 & 97.8 & 4.0 \\
\hline 39 & 85.6 & 87.1 & 92.3 & 97.5 & 99.0 & 4.1 \\
\hline 40 & 86.4 & 87.9 & 93.2 & 98.5 & 100.0 & 4.1 \\
\hline
\end{tabular}


Table 3. Fitted centiles for head circumference in singleton fetuses of pregnant women, in Ampara district

\begin{tabular}{|c|c|c|c|c|c|c|}
\hline \multicolumn{7}{|c|}{ Centiles in millimeters } \\
\hline $\begin{array}{l}\text { Gestational } \\
\text { Age in weeks }\end{array}$ & $\begin{array}{c}5 \text { th } \\
\text { centile }\end{array}$ & $\begin{array}{c}\text { 10th } \\
\text { centile }\end{array}$ & $\begin{array}{l}\text { 50th } \\
\text { centile }\end{array}$ & $\begin{array}{c}\text { 90th } \\
\text { centile }\end{array}$ & $\begin{array}{c}\text { 95th } \\
\text { centile }\end{array}$ & $\begin{array}{l}\text { Standard } \\
\text { deviation }\end{array}$ \\
\hline 12 & 57.8 & 60.6 & 70.4 & 80.2 & 83.0 & 7.7 \\
\hline 13 & 71.4 & 74.3 & 84.3 & 94.3 & 97.1 & 7.8 \\
\hline 14 & 84.9 & 87.7 & 97.9 & 108.1 & 111.0 & 7.9 \\
\hline 15 & 98.1 & 101.0 & 111.4 & 121.8 & 124.7 & 8.1 \\
\hline 16 & 111.0 & 114.0 & 124.6 & 135.2 & 138.1 & 8.2 \\
\hline 17 & 123.7 & 126.8 & 137.5 & 148.3 & 151.3 & 8.4 \\
\hline 18 & 136.2 & 139.3 & 150.2 & 161.2 & 164.3 & 8.5 \\
\hline 19 & 148.3 & 151.5 & 162.6 & 173.8 & 176.9 & 8.7 \\
\hline 20 & 160.2 & 163.4 & 174.7 & 186.0 & 189.3 & 8.8 \\
\hline 21 & 171.7 & 175.0 & 186.5 & 198.0 & 201.3 & 9.0 \\
\hline 22 & 182.9 & 186.2 & 197.9 & 209.6 & 213.0 & 9.1 \\
\hline 23 & 193.8 & 197.1 & 209.0 & 220.9 & 224.3 & 9.3 \\
\hline 24 & 204.3 & 207.7 & 219.8 & 231.9 & 235.3 & 9.4 \\
\hline 25 & 214.4 & 217.8 & 230.1 & 242.4 & 245.9 & 9.6 \\
\hline 26 & 224.1 & 227.6 & 240.1 & 252.5 & 256.1 & 9.7 \\
\hline 27 & 233.4 & 237.0 & 249.6 & 262.3 & 265.9 & 9.9 \\
\hline 28 & 242.2 & 245.9 & 258.7 & 271.6 & 275.2 & 10.0 \\
\hline 29 & 250.7 & 254.4 & 267.4 & 280.4 & 284.1 & 10.2 \\
\hline 30 & 258.6 & 262.4 & 275.6 & 288.8 & 292.6 & 10.3 \\
\hline 31 & 266.1 & 269.9 & 283.3 & 296.8 & 300.6 & 10.5 \\
\hline 32 & 273.1 & 277.0 & 290.6 & 304.2 & 308.0 & 10.6 \\
\hline 33 & 279.6 & 283.5 & 297.3 & 311.1 & 315.0 & 10.8 \\
\hline 34 & 285.6 & 289.5 & 303.5 & 317.5 & 321.5 & 10.9 \\
\hline 35 & 291.0 & 295.0 & 309.2 & 323.4 & 327.4 & 11.1 \\
\hline 36 & 295.9 & 300.0 & 314.3 & 328.7 & 332.7 & 11.2 \\
\hline 37 & 300.2 & 304.3 & 318.9 & 333.4 & 337.5 & 11.4 \\
\hline 38 & 303.9 & 308.1 & 322.8 & 337.6 & 341.7 & 11.5 \\
\hline 39 & 307.0 & 311.2 & 326.2 & 341.1 & 345.3 & 11.6 \\
\hline 40 & 309.5 & 313.8 & 328.9 & 344.0 & 348.3 & 11.8 \\
\hline
\end{tabular}

Table 4. Fitted centiles for abdominal circumference in singleton fetuses of pregnant women, in Ampara district

\begin{tabular}{|c|c|c|c|c|c|c|}
\hline \multicolumn{7}{|c|}{ Centiles in millimeters } \\
\hline $\begin{array}{c}\text { Gestational } \\
\text { Age in weeks }\end{array}$ & $\begin{array}{c}\text { 5th } \\
\text { centile }\end{array}$ & $\begin{array}{l}\text { 10th } \\
\text { centile }\end{array}$ & $\begin{array}{c}50 t h \\
\text { centile }\end{array}$ & $\begin{array}{l}\text { 90th } \\
\text { centile }\end{array}$ & $\begin{array}{c}\text { 95th } \\
\text { centile }\end{array}$ & Standard deviation \\
\hline 12 & 47.8 & 49.8 & 57.1 & 64.4 & 66.5 & 5.7 \\
\hline 13 & 58.3 & 60.5 & 68.4 & 76.3 & 78.6 & 6.2 \\
\hline 14 & 68.8 & 71.2 & 79.7 & 88.2 & 90.6 & 6.6 \\
\hline 15 & 79.2 & 81.7 & 90.9 & 100.0 & 102.6 & 7.1 \\
\hline 16 & 89.4 & 92.2 & 101.9 & 111.6 & 114.4 & 7.6 \\
\hline 17 & 99.6 & 102.6 & 112.9 & 123.2 & 126.1 & 8.1 \\
\hline 18 & 109.7 & 112.8 & 123.7 & 134.7 & 137.8 & 8.5 \\
\hline 19 & 119.7 & 122.9 & 134.5 & 146.0 & 149.3 & 9.0 \\
\hline 20 & 129.5 & 133.0 & 145.1 & 157.3 & 160.7 & 9.5 \\
\hline 21 & 139.2 & 142.8 & 155.6 & 168.4 & 172.0 & 10.0 \\
\hline 22 & 148.8 & 152.6 & 166.0 & 179.3 & 183.1 & 10.4 \\
\hline 23 & 158.3 & 162.2 & 176.2 & 190.2 & 194.1 & 10.9 \\
\hline 24 & 167.6 & 171.7 & 186.3 & 200.9 & 205.0 & 11.4 \\
\hline 25 & 176.7 & 181.0 & 196.2 & 211.4 & 215.7 & 11.9 \\
\hline 26 & 185.7 & 190.2 & 206.0 & 221.8 & 226.2 & 12.3 \\
\hline 27 & 194.5 & 199.2 & 215.6 & 232.0 & 236.6 & 12.8 \\
\hline 28 & 203.2 & 208.0 & 225.0 & 242.0 & 246.8 & 13.3 \\
\hline 29 & 211.6 & 216.6 & 234.3 & 251.9 & 256.9 & 13.7 \\
\hline 30 & 219.9 & 225.1 & 243.3 & 261.6 & 266.7 & 14.2 \\
\hline 31 & 228.0 & 233.4 & 252.2 & 271.1 & 276.4 & 14.7 \\
\hline 32 & 235.9 & 241.5 & 260.9 & 280.3 & 285.9 & 15.2 \\
\hline 33 & 243.6 & 249.3 & 269.4 & 289.4 & 295.1 & 15.6 \\
\hline 34 & 251.1 & 257.0 & 277.7 & 298.3 & 304.2 & 16.1 \\
\hline 35 & 258.4 & 264.4 & 285.7 & 307.0 & 313.0 & 16.6 \\
\hline 36 & 265.5 & 271.7 & 293.5 & 315.4 & 321.6 & 17.1 \\
\hline 37 & 272.3 & 278.7 & 301.2 & 323.6 & 330.0 & 17.5 \\
\hline 38 & 278.9 & 285.4 & 308.5 & 331.6 & 338.2 & 18.0 \\
\hline 39 & 285.2 & 292.0 & 315.7 & 339.4 & 346.1 & 18.5 \\
\hline 40 & 291.4 & 298.2 & 322.5 & 346.9 & 353.7 & 19.0 \\
\hline
\end{tabular}


Table 5. Fitted centiles for femur length in singleton fetuses of pregnant women, in Ampara district

\begin{tabular}{|c|c|c|c|c|c|c|}
\hline $\begin{array}{l}\text { Gestational } \\
\text { Age in weeks }\end{array}$ & $\begin{array}{c}5 \text { th } \\
\text { centile }\end{array}$ & $\begin{array}{c}\text { 10th } \\
\text { centile }\end{array}$ & $\begin{array}{c}\text { 50th } \\
\text { centile }\end{array}$ & $\begin{array}{c}\text { 90th } \\
\text { centile }\end{array}$ & $\begin{array}{c}\text { 95th } \\
\text { centile }\end{array}$ & $\begin{array}{l}\text { Standard } \\
\text { deviation }\end{array}$ \\
\hline 12 & 4.8 & 5.6 & 8.3 & 11.0 & 11.7 & 2.1 \\
\hline 13 & 7.9 & 8.7 & 11.5 & 14.3 & 15.0 & 2.2 \\
\hline 14 & 11.0 & 11.8 & 14.6 & 17.5 & 18.3 & 2.2 \\
\hline 15 & 14.0 & 14.8 & 17.8 & 20.7 & 21.6 & 2.3 \\
\hline 16 & 16.9 & 17.8 & 20.8 & 23.9 & 24.7 & 2.4 \\
\hline 17 & 19.8 & 20.7 & 23.9 & 27.0 & 27.9 & 2.5 \\
\hline 18 & 22.7 & 23.6 & 26.8 & 30.1 & 31.0 & 2.5 \\
\hline 19 & 25.5 & 26.4 & 29.8 & 33.1 & 34.0 & 2.6 \\
\hline 20 & 28.3 & 29.2 & 32.7 & 36.1 & 37.0 & 2.7 \\
\hline 21 & 31.0 & 32.0 & 35.5 & 39.0 & 40.0 & 2.7 \\
\hline 22 & 33.6 & 34.6 & 38.2 & 41.9 & 42.9 & 2.8 \\
\hline 23 & 36.2 & 37.3 & 41.0 & 44.6 & 45.7 & 2.9 \\
\hline 24 & 38.7 & 39.8 & 43.6 & 47.4 & 48.5 & 3.0 \\
\hline 25 & 41.2 & 42.3 & 46.2 & 50.1 & 51.1 & 3.0 \\
\hline 26 & 43.6 & 44.7 & 48.7 & 52.6 & 53.8 & 3.1 \\
\hline 27 & 45.9 & 47.1 & 51.1 & 55.2 & 56.3 & 3.2 \\
\hline 28 & 48.2 & 49.3 & 53.5 & 57.6 & 58.8 & 3.2 \\
\hline 29 & 50.3 & 51.5 & 55.8 & 60.0 & 61.2 & 3.3 \\
\hline 30 & 52.4 & 53.6 & 58.0 & 62.3 & 63.5 & 3.4 \\
\hline 31 & 54.4 & 55.7 & 60.1 & 64.5 & 65.8 & 3.5 \\
\hline 32 & 56.3 & 57.6 & 62.1 & 66.6 & 67.9 & 3.5 \\
\hline 33 & 58.1 & 59.4 & 64.1 & 68.7 & 70.0 & 3.6 \\
\hline 34 & 59.9 & 61.2 & 65.9 & 70.6 & 71.9 & 3.7 \\
\hline 35 & 61.5 & 62.9 & 67.7 & 72.5 & 73.8 & 3.7 \\
\hline 36 & 63.1 & 64.4 & 69.3 & 74.2 & 75.6 & 3.8 \\
\hline 37 & 64.5 & 65.9 & 70.9 & 75.9 & 77.3 & 3.9 \\
\hline 38 & 65.9 & 67.3 & 72.4 & 77.4 & 78.9 & 4.0 \\
\hline 39 & 67.1 & 68.6 & 73.7 & 78.9 & 80.3 & 4.0 \\
\hline 40 & 68.2 & 69.7 & 75.0 & 80.2 & 81.7 & 4.1 \\
\hline
\end{tabular}

Table 6. Comparison of fetal bi-parietal diameter measurements in current study with the reference chart of Chitty et al [10]

\begin{tabular}{|c|c|c|c|c|c|c|c|c|}
\hline \multirow[b]{2}{*}{$\begin{array}{l}\text { Gestational } \\
\text { Age in weeks }\end{array}$} & \multicolumn{3}{|c|}{ BPD Current Study } & \multicolumn{3}{|c|}{ BPD Chitty et al [10] } & \multicolumn{2}{|c|}{ Difference between the two } \\
\hline & $N$ & Mean & $S D$ & $N$ & Mean & $S D$ & Difference & $\begin{array}{c}\text { Estimated } \\
95 \% \text { CI }\end{array}$ \\
\hline 12 & 27 & 21.0 & 0.2 & 4 & 20.8 & 1.5 & 0.2 & -2.35 to 2.75 \\
\hline 13 & 18 & 24.0 & 0.2 & 17 & 23.6 & 1.3 & 0.4 & -0.94 to 1.74 \\
\hline 14 & 2 & 9 & 28.0 & 0.3 & 17 & 29.1 & 1.9 & $-1.1-2.6$ to 0.4 \\
\hline 15 & 30 & 32.0 & 0.3 & 18 & 31.3 & 2.3 & 0.7 & -1.0 to 2.4 \\
\hline 16 & 21 & 36.0 & 0.2 & 17 & 35.5 & 2.4 & 0.5 & -1.02 to 2.02 \\
\hline 17 & 31 & 39.0 & 0.4 & 17 & 37.9 & 3.1 & 1.1 & -0.94 to 3.14 \\
\hline 18 & 22 & 41.0 & 0.3 & 22 & 42.4 & 2.4 & -1.4 & -3.12 to 0.32 \\
\hline 19 & 19 & 46.0 & 0.5 & 20 & 45.6 & 2.6 & 0.4 & -2.13 to 2.93 \\
\hline 20 & 35 & 50.0 & 0.3 & 23 & 47.4 & 2.7 & 2.6 & 0.94 to 4.26 \\
\hline 21 & 26 & 52.0 & 0.3 & 22 & 51.3 & 2.9 & 0.7 & -0.9 to 2.3 \\
\hline 22 & 28 & 54.0 & 0.2 & 21 & 54.7 & 3.2 & -0.7 & -2.25 to 0.85 \\
\hline 23 & 24 & 58.0 & 0.4 & 23 & 58.0 & 3.9 & 0 & -2.8 to 2.18 \\
\hline 24 & 28 & 61.0 & 0.3 & 22 & 60.5 & 3.7 & 0.5 & -1.52 to 2.52 \\
\hline 25 & 24 & 65.0 & 0.3 & 26 & 64.2 & 3.1 & 0.8 & -0.96 to 2.56 \\
\hline 26 & 26 & 68.0 & 0.3 & 13 & 66. 1 & 2.6 & 2.0 & -0.18 to 4.18 \\
\hline 27 & 25 & 70.0 & 0.3 & 21 & 71.4 & 3.4 & -1.4 & -3.24 to 0.44 \\
\hline 28 & 20 & 72.0 & 0.4 & 30 & 75.0 & 3.3 & -3.0 & -4.96 to -1.04 \\
\hline 29 & 27 & 75.0 & 0.4 & 15 & 78.1 & 2.2 & -3.1 & -5.41 to -0.79 \\
\hline 30 & 17 & 79.0 & 0.3 & 24 & 79.7 & 3.0 & -0.7 & -2.68 to 1.28 \\
\hline 31 & 17 & 81.0 & 0.4 & 19 & 81.9 & 3.2 & -0.9 & -3.27 to 1.47 \\
\hline 32 & 22 & 83.0 & 0.4 & 28 & 84.2 & 3.0 & -1.2 & -3.16 to 0.76 \\
\hline 33 & 23 & 84.0 & 0.4 & 22 & 85.4 & 4.4 & -1.4 & -3.81 to 1.01 \\
\hline 34 & 31 & 85.0 & 0.3 & 21 & 87.6 & 2.6 & -2.6 & -4.29 to -0.91 \\
\hline 35 & 30 & 86.0 & 0.5 & 18 & 89.6 & 3.7 & -3.6 & -6.3 to -0.90 \\
\hline 36 & 23 & 88.0 & 0.4 & 22 & 91.0 & 4.4 & -3.0 & -5.50 to -0.50 \\
\hline 37 & 28 & 90.0 & 0.4 & 17 & 92.9 & 4.2 & -2.9 & -5.43 to -0.37 \\
\hline 38 & 27 & 91.0 & 0.4 & 15 & 94.7 & 4.2 & -3.7 & -6.27 to -1.13 \\
\hline 39 & 12 & 92.0 & 0.4 & 13 & 94.8 & 3.8 & -2.8 & -6.11 to 0.51 \\
\hline 40 & 24 & 94.0 & 0.3 & 10 & 97.8 & 4.5 & -3.8 & -6.62 to -0.98 \\
\hline
\end{tabular}

(The bold text indicates the values that are statistically significant). 
Table 7. Comparison of fetal head circumference measurements in current study with the reference chart of Chitty et al [10]

\begin{tabular}{|c|c|c|c|c|c|c|c|c|}
\hline \multirow[b]{2}{*}{$\begin{array}{l}\text { Gestational } \\
\text { Age in weeks }\end{array}$} & \multicolumn{3}{|c|}{$\begin{array}{l}\text { Head Circumference } \\
\text { Current Study }\end{array}$} & \multicolumn{3}{|c|}{$\begin{array}{c}\text { Head Circumference } \\
\text { Chitty et al [10] }\end{array}$} & \multicolumn{2}{|c|}{ Difference between the two } \\
\hline & $N$ & Mean & $S D$ & $N$ & Mean & $S D$ & Difference & $95 \% C I$ \\
\hline 12 & 27 & 71.1 & 7.4 & 4 & 74.0 & 6.3 & -2.9 & -10.89 to 5.09 \\
\hline 13 & 18 & 85.4 & 8.0 & 17 & 83.9 & 6.9 & 1.5 & -3.65 to 6.65 \\
\hline 14 & 29 & 99.4 & 8.2 & 17 & 102.1 & 6.6 & -2.7 & -7.6 to 2.26 \\
\hline 15 & 30 & 110.5 & 10.2 & 18 & 109.8 & 8.2 & 0.7 & -5.01 to 6.41 \\
\hline 16 & 21 & 125.9 & 7.9 & 17 & 124.7 & 7.0 & 1.2 & -3.77 to 6.17 \\
\hline 17 & 31 & 135.7 & 9.6 & 17 & 133.7 & 10.3 & 2.0 & -3.98 to 7.98 \\
\hline 18 & 22 & 145.6 & 8.4 & 22 & 149.8 & 8.8 & -4.2 & -9.43 to 1.03 \\
\hline 19 & 19 & 162.6 & 9.3 & 20 & 163.0 & 8.3 & -0.4 & -6.11 to 5.31 \\
\hline 20 & 35 & 177.9 & 8.5 & 23 & 170.3 & 10.0 & 7.6 & 2.7 to 12.5 \\
\hline 21 & 26 & 186.6 & 8.9 & 22 & 185.3 & 7.9 & 1.3 & -3.63 to 6.23 \\
\hline 22 & 28 & 194.8 & 7.4 & 21 & 193.8 & 10.3 & 1.0 & -4.08 to 6.08 \\
\hline 23 & 24 & 208.5 & 10.6 & 23 & 206.0 & 11.7 & 2.5 & -4.05 to 9.05 \\
\hline 24 & 28 & 219.7 & 8.5 & 22 & 219.0 & 9.4 & 0.7 & -4.4 to 5.8 \\
\hline 25 & 24 & 233.8 & 11.3 & 26 & 230.0 & 8.4 & 3.8 & -1.83 to 9.43 \\
\hline 26 & 26 & 240.4 & 11.4 & 13 & 240.1 & 10.2 & 0.3 & -7.29 to 7.89 \\
\hline 27 & 25 & 249.6 & 11.6 & 21 & 255.0 & 9.1 & -5.4 & -11.69 to 0.89 \\
\hline 28 & 20 & 256.9 & 9.0 & 30 & 266.7 & 9.6 & -9.8 & -15.24 to -4.36 \\
\hline 29 & 27 & 267.3 & 13.3 & 15 & 279.2 & 6.8 & -11.9 & -19.35 to -4.45 \\
\hline 30 & 17 & 280.2 & 10.4 & 24 & 284.7 & 9.3 & -4.5 & -10.23 to 1.23 \\
\hline 31 & 17 & 286.2 & 12.8 & 19 & 294.5 & 10.1 & -8.3 & -16.07 to -0.53 \\
\hline 32 & 22 & 292.3 & 13.0 & 28 & 300.0 & 10.9 & -7.7 & -14.5 to -0.9 \\
\hline 33 & 23 & 299.7 & 12.7 & 22 & 306.6 & 12.7 & -6.9 & -14.54 to 0.74 \\
\hline 34 & 31 & 302.5 & 8.7 & 21 & 311.8 & 10.3 & -9.3 & -14.62 to -3.98 \\
\hline 35 & 30 & 305.7 & 12.7 & 18 & 317.3 & 13.1 & -11.6 & -19.31 to -3.89 \\
\hline 36 & 23 & 310.6 & 12.6 & 22 & 327.0 & 13.6 & -16.4 & -24.28 to -8.52 \\
\hline 37 & 28 & 320.0 & 11.2 & 17 & 329.3 & 11.5 & -9.3 & -14.83 to -3.77 \\
\hline 38 & 27 & 322.7 & 8.5 & 15 & 333.1 & 18.1 & -10.3 & -18.54 to -2.06 \\
\hline 39 & 12 & 325.5 & 8.8 & 13 & 335.1 & 11.8 & -9.6 & -18.27 to -0.93 \\
\hline 40 & 24 & 331.3 & 10.2 & 10 & 341.9 & 18.6 & -10.6 & -20.66 to -0.54 \\
\hline
\end{tabular}

The bold text indicates the values that are statistically significant.

Table 8. Comparison of fetal abdominal circumference measurements in current study with the reference chart of Chitty et al [11]

\begin{tabular}{|c|c|c|c|c|c|c|c|c|}
\hline \multirow[b]{2}{*}{$\begin{array}{c}\text { Gestational } \\
\text { Age in weeks }\end{array}$} & \multirow[b]{2}{*}{$N$} & \multicolumn{2}{|c|}{$\begin{array}{c}\text { Abdominal circumference } \\
\text { Current Study }\end{array}$} & \multicolumn{2}{|c|}{$\begin{array}{c}\text { Abdominal } \\
\text { circumference Chitty } \\
\text { et al [11] }\end{array}$} & \multirow[b]{2}{*}{$S D$} & \multicolumn{2}{|c|}{ Difference between the two } \\
\hline & & Mean & $S D$ & $N$ & Mean & & Difference & $95 \% C I$ \\
\hline 12 & 27 & 58.9 & 6.4 & 4 & 58.5 & 3.7 & 0.4 & -6.37 to 7.17 \\
\hline 13 & 18 & 68.8 & 5.5 & 11 & 68.5 & 6.0 & 0.3 & -4.17 to 4.77 \\
\hline 14 & 29 & 79.3 & 7.3 & 10 & 80.1 & 5.6 & -0.8 & -5.95 to 4.35 \\
\hline 15 & 30 & 92.2 & 9.6 & 10 & 92.2 & 7.4 & 0 & -6.75 to 6.75 \\
\hline 16 & 21 & 103.1 & 9.8 & 8 & 105.4 & 8.3 & -2.3 & -10.34 to 5.74 \\
\hline 17 & 31 & 111.2 & 9.0 & 11 & 112.0 & 4.4 & -0.8 & -6.54 to 4.94 \\
\hline 18 & 22 & 121.5 & 8.2 & 16 & 123.3 & 10.5 & -1.8 & -7.95 to 4.35 \\
\hline 19 & 19 & 133.9 & 7.0 & 15 & 137.2 & 9.0 & -3.3 & -8.88 to 2.28 \\
\hline 20 & 35 & 148.3 & 10.6 & 14 & 144.9 & 8.8 & 3.4 & -3.05 to 9.85 \\
\hline 21 & 26 & 153.6 & 9.0 & 17 & 159.2 & 8.9 & -5.6 & -11.24 to 0.04 \\
\hline 22 & 28 & 160.2 & 13.0 & 16 & 163.8 & 8.8 & -3.6 & -10.96 to 3.78 \\
\hline 23 & 24 & 177.0 & 11.2 & 17 & 175.9 & 11.4 & 1.0 & -6.23 to 8.23 \\
\hline 24 & 28 & 186.1 & 10.8 & 12 & 187.9 & 10.2 & -1.8 & -9.22 to 5.62 \\
\hline 25 & 24 & 200.4 & 12.5 & 12 & 195.8 & 9.1 & 4.6 & -3.67 to 12.87 \\
\hline 26 & 26 & 207.9 & 10.0 & 6 & 209.5 & 12.5 & -1.6 & -11.27 to 8.07 \\
\hline 27 & 25 & 214.0 & 11.1 & 17 & 218.1 & 11.0 & -4.1 & -11.13 to 2.93 \\
\hline 28 & 20 & 221.0 & 9.8 & 17 & 234.3 & 15.9 & -13.3 & -21.97 to -4.63 \\
\hline 29 & 27 & 232.6 & 15.8 & 6 & 242.8 & 13.3 & -10.2 & -24.4 to 4.00 \\
\hline 30 & 17 & 244.2 & 12.0 & 10 & 246.2 & 18.4 & -2.0 & 14.01 to 10.01 \\
\hline 31 & 17 & 256.7 & 14.4 & 13 & 267.5 & 21.5 & -10.8 & -24.23 to 2.63 \\
\hline 32 & 22 & 266.8 & 16.0 & 20 & 271.1 & 12.0 & -5.3 & -14.19 to 3.56 \\
\hline 33 & 23 & 268.5 & 18.1 & 13 & 290.0 & 22.7 & -21.5 & -35.49 to -7.51 \\
\hline 34 & 31 & 279.3 & 14.3 & 21 & 289.5 & 18.0 & -10.2 & -19.22 to -1.18 \\
\hline 35 & 30 & 281.6 & 13.5 & 14 & 300.4 & 15.2 & -18.8 & -27.98 to -9.62 \\
\hline 36 & 23 & 297.5 & 14.4 & 19 & 307.4 & 20.9 & -9.9 & -20.94 to 1.14 \\
\hline 37 & 28 & 305.4 & 16.4 & 14 & 317.0 & 18.8 & -11.6 & -22.99 to -0.21 \\
\hline 38 & 27 & 301.4 & 19.9 & 15 & 319.8 & 22.4 & -18.4 & -31.94 to -4.86 \\
\hline 39 & 12 & 319.1 & 26.2 & 15 & 330.6 & 18.1 & -11.5 & -29.08 to 6.08 \\
\hline 40 & 24 & 322.8 & 20.0 & 12 & 344.2 & 23.0 & -21.4 & -36.5 to -6.30 \\
\hline
\end{tabular}

The bold text indicates the values that are statistically significant. 
Table 9. Comparison of fetal femur length in current study with the reference chart of Chitty et al [9]

\begin{tabular}{|c|c|c|c|c|c|c|c|c|}
\hline \multirow[b]{2}{*}{$\begin{array}{l}\text { Gestational } \\
\text { Age in weeks }\end{array}$} & \multirow[b]{2}{*}{$N$} & \multicolumn{2}{|c|}{$\begin{array}{l}\text { Femur Length } \\
\text { Current Study }\end{array}$} & \multirow[b]{2}{*}{$N$} & \multicolumn{2}{|c|}{$\begin{array}{l}\text { Femur Length } \\
\text { Chitty et al [9] }\end{array}$} & \multicolumn{2}{|c|}{ Difference in two groups } \\
\hline & & Mean & $S D$ & & Mean & $S D$ & Difference & $95 \% C I$ \\
\hline 12 & 27 & 9.3 & 2.2 & 4 & 8.5 & 0.6 & 0.8 & -1.49 to 3.09 \\
\hline 13 & 18 & 11.8 & 2.6 & 17 & 11.0 & 1.6 & 0.8 & -0.71 to 2.31 \\
\hline 14 & 29 & 13.6 & 2.4 & 17 & 14.1 & 1.9 & -0.5 & -1.87 to 0.87 \\
\hline 15 & 30 & 17.3 & 1.7 & 18 & 17.1 & 2.4 & 0.2 & -0.99 to 1.39 \\
\hline 16 & 21 & 20.7 & 3.6 & 17 & 20.5 & 2.2 & 0.2 & -9.97 to 10.11 \\
\hline 17 & 31 & 23.7 & 2.5 & 17 & 22.7 & 2.2 & 1.0 & -0.46 to 2.46 \\
\hline 18 & 22 & 26.8 & 1.9 & 22 & 26.9 & 2.4 & -0.1 & -1.4 to 1.2 \\
\hline 19 & 19 & 29.7 & 2.0 & 20 & 29.5 & 2.1 & 0.2 & -1.12 to 1.52 \\
\hline 20 & 35 & 33.4 & 2.6 & 23 & 32.2 & 2.2 & 1.2 & -0.13 to 2.53 \\
\hline 21 & 26 & 36.3 & 3.7 & 22 & 35.4 & 2.6 & 0.9 & -1.00 to 2.8 \\
\hline 22 & 28 & 37.7 & 3.1 & 21 & 37.2 & 2.5 & 0.5 & -1.16 to 2.16 \\
\hline 23 & 24 & 41.5 & 2.4 & 23 & 40.6 & 3.2 & 0.9 & -0.75 to 2.55 \\
\hline 24 & 28 & 43.9 & 3.3 & 22 & 43.5 & 2.3 & 0.4 & -1.26 to 2.06 \\
\hline 25 & 24 & 46.3 & 2.5 & 26 & 46.1 & 3.6 & 0.2 & -1.56 to 1.96 \\
\hline 26 & 26 & 47.8 & 2.7 & 13 & 46.9 & 2.2 & 0.9 & -0.86 to 2.66 \\
\hline 27 & 25 & 50.2 & 3.8 & 21 & 50.2 & 2.7 & 0 & -1.99 to 1.99 \\
\hline 28 & 20 & 53.4 & 2.9 & 30 & 52.4 & 2.9 & 1.0 & -0.84 to 2.84 \\
\hline 29 & 27 & 55.7 & 3.6 & 15 & 56.3 & 1.8 & -0.6 & -2.23 to 1.03 \\
\hline 30 & 17 & 59.4 & 2.6 & 24 & 56.0 & 2.9 & 3.4 & 1.62 to 5.18 \\
\hline 31 & 17 & 59.7 & 2.9 & 19 & 59.7 & 3.7 & 0 & -2.27 to 2.27 \\
\hline 32 & 22 & 63.7 & 2.5 & 28 & 61.3 & 3.0 & 2.4 & 0.88 to 3.92 \\
\hline 33 & 23 & 63.8 & 4.2 & 22 & 62.8 & 2.9 & 1.0 & -1.2 to 3.2 \\
\hline 34 & 31 & 66.6 & 3.8 & 21 & 64.3 & 2.5 & 2.3 & 0.41 to 4.19 \\
\hline 35 & 30 & 67.3 & 3.7 & 18 & 66.2 & 3.3 & 1.1 & -1.03 to 3.13 \\
\hline 36 & 23 & 69.3 & 4.2 & 22 & 68.3 & 3.1 & 1.0 & -1.22 to 3.22 \\
\hline 37 & 28 & 70.9 & 4.4 & 17 & 69.9 & 3.0 & 1.0 & $1-145$ to 3.45 \\
\hline 38 & 27 & 72.0 & 4.8 & 15 & 70.8 & 3.6 & 1.2 & -1.67 to 4.07 \\
\hline 39 & 12 & 73.9 & 2.6 & 13 & 71.7 & 3.0 & 2.2 & -0.08 to 4.48 \\
\hline 40 & 24 & 75.3 & 4.6 & 10 & 74.7 & 4.0 & 0.6 & -2.8 to 4.0 \\
\hline
\end{tabular}

The bold text indicates the values that are statistically significant.

In contrast to our study which is a cross sectional study, the "INTERGROWTH- 21st" was carried out as a multi-centric longitudinal study. The 50th centile values for BPD, HC, AC and FL at 40 weeks of gestation in the INTERGROWTH- 21st study were 94.89, 333.94, 349.8 and 72.13 respectively, whereas the values of the same parameters in our study are 93.2, 328.9, 322.5 and 75 respectively [19]. However it is not possible to compare and contrast charts based on longitudinal data with charts based on cross sectional data.

The main strength of our study is that we adopted strict inclusion criteria to select a population with normal nutritional and health status and minimal environmental constraints on fetal growth [20]. It has been suggested that once the maternal social, physical and environmental characteristics are optimised, fetal growth would be similar in different populations [19]. Our study is further strengthened because the differences between the local fetal biometry and the reference charts have been observed in pregnant women with normal BMI. Furthermore, all study participants had their pregnancies dated by first trimester ultra-sonographic fetal CRL measurement. In contrast to pregnancies that are dated by menstrual dating method, which tend to overestimate the length of gestation, resulting in term weights being spread across a wider gestational age range, routine ultrasound-dated birth weight charts demonstrate no such flattening at term [21]. Our chart too did not show any flattening towards term.

In our study, we have used statistical methods that pay due attention to the increasing variability of the measurements with increasing gestational age in the construction of the centile charts, and we have carefully assessed the goodness of fit of the models obtained [14].

Even though a well-trained, skillful sonologist obtained the fetal biometry measurements, the main limitations of our study was failure to check for intra observer variations in obtaining the fetal biometric measurements, not adequately blinding the sonologist and not checking the accuracy and precision of the sonologist. It has been recommended that ideally at least two measurements should be obtained at each assessment, and that the sonologist should be blinded to the measurements with the measurements not appearing on the screen [22, 23].

Furthermore the accuracy and precision of the sonologist should be checked in comparison to an expert. It is generally recommended that the Technical Error of Measurement (TEMs) should be calculated, and for a sonologist to be accredited to obtain such measurements 
for research purposes, the sonologist should have a TEM $<$ twice that of an expert [22-24].

It has been shown that both inter- and intra-observer variability of fetal biometry increases with increasing size and, consequently, gestational age. This trend is known to be more pronounced for $\mathrm{HC}$ and AC than for FL [18]. However, studies have also shown good intraand inter observer reproducibility of ultrasound measurements of fetal biometry [25-27]. In the INTERGROWTH21st study centiles were derived from serial (longitudinal) measurements from each fetus [19]. Such data are necessary for the development of centiles for growth, but they are not suitable for developing centiles of size.

Gestation specific centile charts for "size”, are derived from cross sectional studies with fetal biometry measurements obtained from its study population comprising of pregnant women of different gestational ages. However gestation specific centile charts for "growth" are derived from longitudinal studies, where a study population is followed up while obtaining fetal biometry measurements at regular intervals during their pregnancies. In clinical practice, "size charts” derived from a cross sectional study helps to diagnose SGA and LGA promptly with a single set of fetal biometry measurements (HC, AC, BPD and FL). In contrast, growth charts derived from a longitudinal study requires serial measurements over a certain period of time to make the diagnosis. Because serial measurements on an individual fetus are highly correlated, the effective sample size in such a study is large. Therefore recruitment of a large sample size is not pragmatic (nearly 20,000 observations) in a single study site [19].

Simplified charts for fetal ultrasound biometry are invaluable as it is difficult to have individually customized charts globally, especially in low resource setting. However, it is important to note that only 714 (22\%) pregnant women presenting for antenatal care in the four $\mathrm{MOH}$ areas of Ampara fulfilled the inclusion criteria for our study. Therefore a prospective multicenter study, with adequate and appropriate sampling methods, incorporating all healthy women, irrespective of BMI, with no medical or obstetric complications, adhering to all the standards required to obtain valid ultra sound measurements of fetal biometry, should be carried out. This would be more representative of the country and enable national nomograms to be developed for different BMI groups in Sri Lanka.

In conclusion, our study has shown that ultrasound fetal biometry of singleton fetuses in pregnant women with normal nutritional and health status in the Ampara district were significantly different in the third trimester, from a routinely used reference chart.

\section{Acknowledgement}

We acknowledge the guidance and support of Professor Malik Goonewardene in presentation of the results and editing this manuscript.

\section{Conflicts of interest}

There are no conflicts of interest.

\section{References}

1, Dudley NJ. A systematic review of the ultrasound estimation of fetal weight. Ultrasound Obstet Gynecol 2005; 25 : 80-9.

2. Johnsen SL, Rasmussen S, Sollien R, Kiserud T. Accuracy of second trimester fetal head circumference and biparietal diameter for predicting the time of spontaneous birth. $J$ Perinat Med 2006; 34: 367-70.

3. Scott N, Rudy E. Assessment of Gestational Age by Ultrasound. The global library of women's medicine, 2008.http://www.glowm.com/section_view/heading/ Assessment\%20of\%20Gestational\%20Age\%20by\%20 Ultrasound/item/206 (accessed on April 29, 2016).

4. Werner H, Fazecas T, Guedes B, et al. Intrauterine Zika virus infection and microcephaly: perinatal imaging correlations with 3D virtual physical models. Ultrasound Obstet Gynecol 2016; 47: 657-60

5. Royal College of Obstetricians and Gynaecologists. Green top Guideline No. 31, The Investigation and Management of the Small for Gestational Age Fetus. 2013.

6. Krakow D, Lachman RS, Rimoin DL. Guidelines for the prenatal diagnosis of fetal skeletal dysplasias. Genet Med 2009; 11: 127-33.

7. Njoku C, Emechebe C, Odusolu P, Abeshi S, Chukwu C, Ekabua J. Determination of accuracy of fetal weight using ultrasound and clinical fetal weight estimations in Calabar South, South Nigeria. Int Sch Res Notices 2014.

8. Sharma N, Srinivasan KJ, Sagayaraj MB, Lal DV. Foetal weight estimation methods-clinical, sonographic and MRI imaging. Int J Sci Res Pub 2014; 4: 2250-3153.

9. Chitty LS, Altman DG, Henderson A, Campbell S. Charts of fetal size: 4. Femur length. BJOG 1994; 101: 132-5.

10. Chitty LS, Altman DG, Henderson A, Campbell S. Charts of fetal size. 2: Head measurements. BJOG 1994; 101: 35-43.

11. Chitty LS, Altman DG, Henderson A, Campbell S. Charts of fetal size. 3: Abdominal circumference. BJOG 1994; 101: 125-31.

12. Chitty LS, Altman DG. Charts of fetal size: limb bones. BJOG 2002; 109: 919-29.

13. Salomon LJ, Alfirevic Z, Berghella V, et al. Practice guidelines for performance of the routine mid-trimester fetal ultrasound scan. Ultrasound Obstet Gynecol 2011; 37: 116-26.

14. Altman DG, Chitty LS. Charts of fetal size: 1 . Methodology. BJOG 1994; 101: 129-34. 
15. Katz J, Lee ACC, Kozuk Ni, et al. Small for Gestational Age Preterm Birth Working Group. Mortality risk in preterm and small for gestational age infants in low income and middle income countries: a pooled country analysis. Lancet 2013; 382: 417-25.

16. Airede AI. Birth weight of Nigerian newborn infants - a review. West Afr J Med 1995; 14: 116-20.

17. Shittu AS, Kuti O, Orji EO, et al. Clinical versus sonographic estimation of foetal weight in southwest Nigeria. $J$ Health Popul Nutr 2007; 25: 14-23.

18. Sarris I, Ioannou C, Chamberlain P, et al. Intra- and interobserver variability in fetal ultrasound measurements. Ultrasound Obstet Gynecol 2012; 39: 266-73.

19. Papageorghiou AT, Ohuma EO, Altman DG, et al. International Fetal and Newborn Growth Consortium for the 21st Century (INTERGROWTH-21st). International standards for fetal growth based on serial ultrasound measurements: the Fetal Growth Longitudinal Study of the INTERGROWTH-21st Project. Lancet 2014; 384: 869-87.

20. Villar J, Altman DG, Purwar M, et al. The objectives, design and implementation of the INTERGROWTH - 21st Project. BJOG 2013; 120 (s2): 9-26.

21. Wilcox M, Gardosi J, Mongelli M, et al. Birth weight from pregnancies dated by ultrasonography in a multicultural British population. BMJ 1993; 307: 588-91.
22. Ioannou C, Talbot K, Ohuma E, et al. Systematic review of methodology used in ultrasound studies aimed at creating charts of fetal size. BJOG 2012; 119: 1425-39.

23. Papageorghiou AT, Sarris I, Ioannou C, et al. Ultrasound methodology used to construct the fetal growth standards in the INTERGROWTH-21st Project. BJOG 2013; 120 (s2): 27-32.

24. Neufeld LM1, Wagatsuma Y, Hussain R, Begum M, Frongillo EA. Measurement error for ultrasound fetal biometry performed by paramedics in rural Bangladesh. Ultrasound Obstet Gynecol 2009; 34: 387-94.

25. Perni SC, Chervenak FA, Kalish RB, et al. Intraobserver and interobserver reproducibility of fetal biometry. Ultrasound Obstet Gynecol 2004; 24: 654-8.

26. Yang F, Leung KY, Lee YP, Chan HY, Tang MH. Fetal biometry by an inexperienced operator using two- and threedimensional ultrasound. Ultrasound Obstet Gynecol 2010; 35: 566-71.

27. Nakai A, Oya A. Accuracy and reproducibility of ultrasound measurements in obstetric management. Gynecol Obstet Invest 2002; 54: 31-6.

28. Dias T, Abeykoon S, Kumarasiri S. Symphysis-pubis fundal height charts to assess fetal size in women with a normal body mass index. Ceylon Med J 2016; 61: 106-12. 